\title{
Change Detection Without Awareness: Do Explicit Reports Underestimate the Representation of Change in the Visual System?
}

\author{
Diego Fernandez-Duque \\ Psychology Department, University of Oregon, Eugene, USA \\ Ian M. Thornton \\ Cambridge Basic Research, Nissan Research and Development, Inc., \\ Cambridge, MA, USA
}

\begin{abstract}
Evidence from many different paradigms (e.g. change blindness, inattentional blindness, transsaccadic integration) indicate that observers are often very poor at reporting changes to their visual environment. Such evidence has been used to suggest that the spatio-temporal coherence needed to represent change can only occur in the presence of focused attention. In four experiments we use modified change blindness tasks to demonstrate (a) that sensitivity to change does occur in the absence of awareness, and (b) this sensitivity does not rely on the redeployment of attention. We discuss these results in relation to theories of scene perception, and propose a reinterpretation of the role of attention in representing change.
\end{abstract}

\section{INTRODUCTION}

Observers are very often unaware of large changes in their visual environment until attention is drawn directly to those objects and/or dimensions that are being transformed. Such "change blindness" occurs for many types of transformation, including changes of object colour, location, and identity (e.g. Levin \& Simons, 1997, Rensink, O' Regan, \& Clark, 1997), appearing to be equally prevalent in situations in which stimuli are static images (e.g. Rensink, O' Regan \& Clark, this issue), animation sequences (e.g. Wallis \& Bülthoff, this issue), movie clips (e.g. Levin \& Simons, 1997) or even real-world interaction s

All correspondence should be addressed to I.M. Thornton, Nissan CBR, 4 Cambridge Center, Cambridge, MA 02142, USA. Email: ian@ pathfinder.cbr.com

The authors contributed equally to this research, and authorship order was determined arbitrarily. Thanks are extended to Jack Beusmans, Arien Mack, Mike Posner, Ron Rensink, Adriane Seiffert, and an anonymous reviewer for commenting on an earlier draft of this manuscript. 
(Simons \& Levin, 1997). The effect is robust across both short (e.g. Pashler, 1988; Simons, 1996) and long display durations (e.g. Bridgeman, Hendry, \& Stark, 1975; Rensink et al., 1997), and can occur whenever the local transients which usually mark such changes are disrupted by saccades (e.g. Grimes, 1996; Henderson, 1997), blinks (O'Regan, Deubel, Clark, \& Rensink, this issue), global blanking fields (e.g. Rensink et al., 1997), movie cuts (Levin \& Simons, 1997), or distracting local transient masks (Rensink, et al., this issue).

A general conclusion from this body of work is that attention is necessary for detecting change (Rensink et al., 1997). Similarly, such findings have been used as evidence that, outside the range of focal attention, our mental representations of the visual world are extremely volatile. Specifically, it has been argued that in the absence of attention our view of the world is so unstable as to be unable to support the representation of change over time (Rensink, this issue; Horowitz \& Wolfe, 1998).

In this model of perception, attention is seen as the glue that binds objects across both space and time (Treisman, 1988). Such glue allows us to create and maintain a small number of coherent "object files", structures that can be updated to reflect change and that provide us with a detailed window on the world at any given moment in time (Kahneman, Treisman, \& Gibbs, 1992). Recent theories of scene perception suggest that it is the dynamic allocation of these attentional objects, rather than the construction of a single, static representation of the entire scene, that gives rise to our subjective impression of a rich, detailed perceptual world (e.g. Rensink, this issue).

The most important assertion to come out of this line of research is that spatio-temporally coherent representations can only exist in the presence of focused attention (e.g. Rensink, this issue). Evidence for the lack of coherent representation s beyond the focus of attention, however, has come mainly from experiments that require observers to both explicitly detect and explicitly respond to a change. As techniques to assess the visual system's ability to represent change, such explicit measures may be quite insensitive in that they rely exclusively on conscious awareness (Marcel, 1983a, 1983b). As there is considerable evidence from other domains of psychology that the mind can represent and process information outside the focus of attention and beneath the level of conscious awareness, it is thus possible that we may be systematically underestimating our ability to process and represent change at some level(s) in the visual system (Hayhoe, this issue; Simons \& Levin, 1997).

The distinction between implicit and explicit measures of behaviour is now very well established. For instance, classic work with human amnesic patients demonstrated that encoding and retrieval of information can proceed independently of explicit awareness (e.g. Milner, Corkin, \& Teuber, 1968; Warrington $\&$ Weiskrantz, 1968). Also, lesions in primary visual areas can lead to a condition known as "blindsight" in which patients lose visual awareness in certain parts of the visual field, but retain the ability to correctly "guess" or respond to 
the stimulus properties (e.g. size, velocity, orientation) of objects presented at these apparently "blind" locations (Weiskrantz, 1986).

There is also evidence that stimuli can be processed by the visual system even when the lack of awareness is due to an attention deficit rather than a visual deficit, as with the case of unilateral neglect. Although stimuli presented to a neglected visual field usually fails to reach awareness, such stimuli can nevertheless be used for figure-ground segregation and can also contribute to geometric illusions (Driver, Baylis, \& Rafal, 1992; Ro \& Rafal, 1996).

Unconscious information processing is also found in normal subjects. Priming studies have shown that both sub-threshold (e.g. Bar \& Biederman, 1998; Marcel, 1983a) and supra-threshold (e.g. DeSchepper \& Treisman, 1996) stimuli can have substantial effects on subsequent behaviour even though explicit encoding was reduced or absent. Most relevant to the current issues, a number of perceptual studies have recently demonstrated that objects or events in the visual field can influence behaviour even when those objects or events are not consciously detected by the observer (Chen, 1998; Graves \& Jones, 1992; Kolb \& Braun, 1995; Mack \& Rock, 1998; McCormick, 1997; Moore \& Egeth, 1997). For example, Kolb and Braun (1995) were able to induce a form of "blindsight" in normal subjects by presenting displays in which a target region was simultaneously masked either by competing motion elements or binocularly rivalrous elements. Localization of the target region remained well above chance in these displays, but confidence ratings were uncorrelated with this performance, suggesting that observers believed themselves to be guessing. Moore and Egeth (1997) used a variant of the inattentional blindness paradigm (Mack \& Rock, 1998) to demonstrate that judgements of line length could be influenced by the configuration of background elements even though on the critical trials observers were unable to report or recognize the presented configuration.

In four experiments, we examine whether changes which are not explicitly detected by an observer can nonetheless influence subsequent behaviour. Evidence for such implicit change detection would have important implications for theories of object and scene perception, particularly with respect to the role that attention might play in establishing and maintaining perceptual representations.

\section{EXPERIMENT 1}

Even when observers explicitly report not seeing a change in a visual display, can such change still influence behaviour? To address this question we combined a standard change detection task with a two-alternative forced choice (2AFC) response method. Each trial began with a change detection sequence in which observers were presented with two brief 16-rectangle displays, separated by a blank screen (see Figure 1). The duration of the rectangle displays 


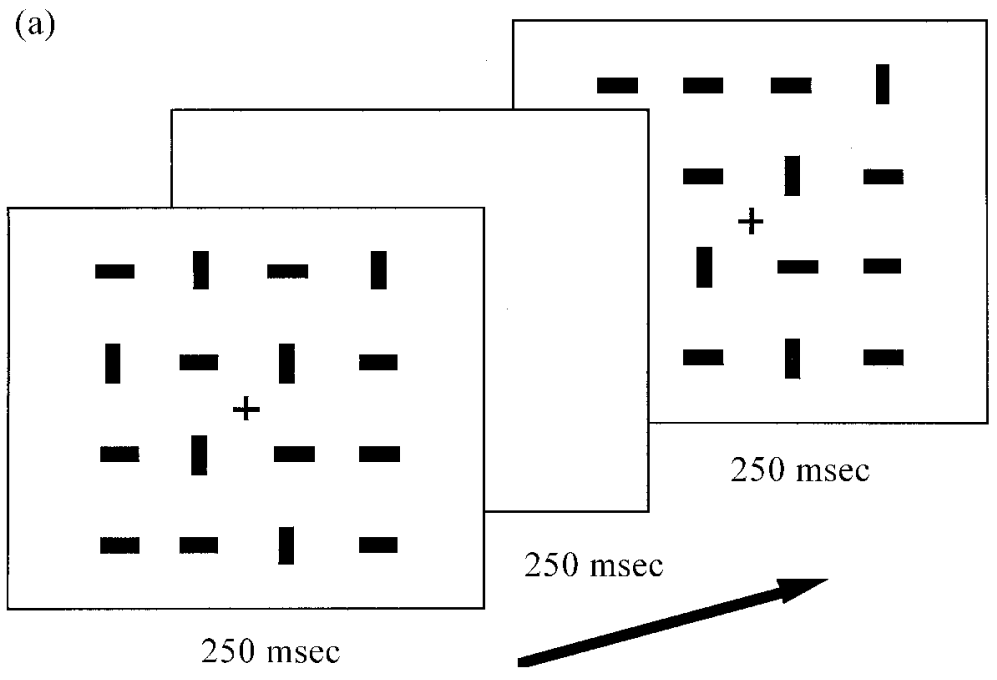

(b)

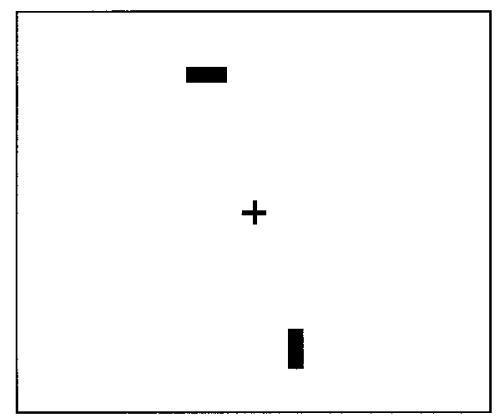

(c)

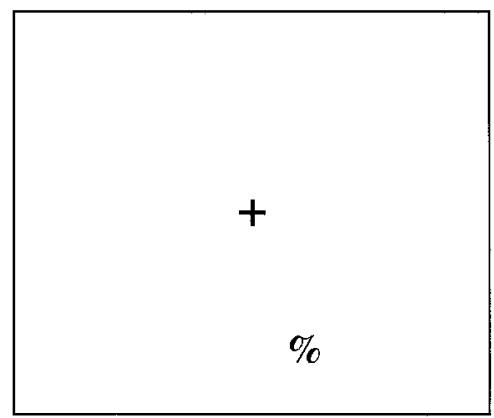

FIG. 1. Schematic representation of the layout and timeline for the stimuli used in Experiments 1 and 2. All displays were centred in the middle of the monitor screen without a visible border, and the background and blank screen were grey rather than white. (a) The change detection sequence shown in the initial portion of each trial. The two rectangle frames are identical except for a single object which changes orientation during the blank ISI. Change was equiprobable at any location and here involves the rectangle in the top row, second column from the left. (b) Experiment 1 response display. This followed the second change sequence display after a 0 ISI. Participants moved the central fixation point using the mouse and clicked on the rectangle that they thought most likely to have changed. The correct selection in this case would be the top rectangle. (c) Experiment 2 response display. This followed the second change sequence display after a 0 ISI. A single target item appeared which, in separate blocks, could be one of two alphanumeric characters ( $\&$ or $\%$ ) or one of two coloured rectangles (red or green). Participants made a speeded response using one of two marked keys. The position of the target could overlap either with the change location (valid cue) or the distractor location (invalid cue, shown). 
and the blank interstimulus interval (ISI) was fixed at $250 \mathrm{msec}$. The first display contained eight horizontal and eight vertical rectangles and the second display differed only in that the orientation of one of these rectangle changed during the ISI. This "changed" object, which could appear at any random location, along with its diametrically opposite "distractor" object, were then presented to observers (see Figure 1b) who made a 2AFC decision as to which of the two rectangles they thought most likely to have changed. Participants were told to guess if they were unsure.

Following the target selection, participants indicated whether they had seen a change or not by pressing one of two marked keys. In separate blocks of trials the precise instructions given to the participant for making the aware/unaware decision was varied. In the conservative block, participants were told to choose an aware response only if they had actually seen the selected rectangle change orientation. In the liberal block, they were told to choose aware if they had noticed or felt any change at all between the first two displays. The order of these two blocks of trials was counterbalanced across participants.

Previous research with such brief change detection displays (e.g. Pashler, 1988; Phillips, 1974; Simons, 1996) suggests that, in general, observers should be quite poor at explicitly reporting the change in orientation between the first and second display. Clearly the use of liberal rather than conservative aware instructions should increase the proportion of trials in which observers are prepared to say they experienced a change.

Of most interest here, however, is whether the report of being unaware accurately indicates that change has not been represented. To assess this, we examined accuracy and reaction time of target selection on those trials in which observers report being unaware of any change. If the explicit awareness response is an accurate reflection of what was being represented in the visual system, then observers should perform at chance when selection is accompanied by an unaware response and reaction time to select target or distractor items should not differ.

${ }^{1}$ Catch trials were not included in these experiments. Catch trials allow signal detection analysis, which is a useful tool in separating perceptual effect from criterion biases. However, we decided not use catch trials, for several reasons: (1) we expected change detection to be quite hard, and worried that including catch trials would make detection rate so low that observers would disengage from the task. (2) we wanted to maximize our power by including as many trials as possible in which a change occurred. (3) we instructed subjects to use a liberal or conservative criterion for awareness. Finding above-chance performance for unaware trials when subjects are liberal in reporting awareness would serve a similar function to catch trials in ruling out criterion biases as a single cause of the effect. 


\section{Method}

Participants. Twenty University of Oregon students took part in this study for partial course credit. All participants were right handed, had normal or corrected to normal vision and were naive as to the purpose of the study. Unless noted, this applies also to the other studies.

Equipment. Stimuli display and response collection were carried out on a Power Macintosh 7200 attached to a standard 15" $(27 \times 20 \mathrm{~cm})$ RGB monitor with a frame rate of $75 \mathrm{~Hz}$ and a screen resolution of $832 \times 624$ pixels. Software was custom written using Think $C$ version 7.0. Many of the routines were based on work by Steinman and Nawrot (1992), Pelli and Zhang (1991), and Rensink (1990). A chin rest was used to fix the subjects' viewing distance approximately $40 \mathrm{~cm}$ from the monitor. Details of equipment did not vary between experiments and will not be reported again.

Stimuli. Rectangles were drawn in black on a uniform grey background. The same grey level was also used for the blank field which separated the two change displays. Each rectangle measured $10 \times 30$ pixels, which subtended $0.46^{\circ} \times 1.38^{\circ}$ visual angle (VA). Rectangles were organized around a central fixation point in a $4 \times 4$ grid, with the centre of each rectangle separated from its neighbour by 70 pixels or $3.25^{\circ} \mathrm{VA}$ (see Figure 1). The entire grid thus subtended approximately $11^{\circ} \times 11^{\circ}$. Eccentricity from the central fixation varied with position in the grid. The centres of the nearest four rectangles were approximately $2.3^{\circ}$, the four corner rectangles were $7.9^{\circ}$, and the remaining eight rectangles were $5.8^{\circ}$ eccentric from fixation. In the first display of each trial, half the rectangles were horizontal and half were vertical, the distribution of orientation by position being randomized for each trial. In the second display, the orientation of one rectangle was switched by having it rotate $90^{\circ}$ about its centre point. This change could take place at any location with equal probability, with each rectangle serving as the target on 16 trials per block ( 256 trials per block) in an order that was randomized separately for each participant. The distractor item for a given changing rectangle was fixed and consisted of the rectangle that was at an equal distance from fixation along the main diagonal (see Figure 1b). This ensured that the changing item and the distractor were always in opposite quadrants, that is, across both the left-right meridian and the up-down equator, and were always at an equal eccentricity.

Procedure. Participants were asked to fixate a central cross during the first two rectangle displays, but were allowed to move their eyes at all other times. They were informed that on some trials, a change of orientation would occur and that they should try to notice such a change. During the third display, when only the two test rectangles were visible, the task of the participant was to move 
the central fixation point with the mouse and click on the rectangle that they thought most likely to have changed orientation. The mouse was always located in front of the participant, centred relative to the middle of the screen. After illustrating the general procedure, participants were given a number of practice trials and then completed a block of 256 trials, followed by a second block in which more conservative (or liberal) criterion for awareness was adopted.

Participant Selection. When participants failed to select the correct rectangle in trials in which they reported having seen a change, it is likely that they were not co-operating or did not understand the instructions. Thus, we established a 70\% accuracy criterion for aware trials, and replaced any participant who failed to reach this level of performance. The same criterion was used in Experiment 3. Two participants were replaced overall.

\section{Results and Discussion}

As expected, observers were poor at explicitly reporting changes when they were instructed to use conservative awareness criteria, indicating that they saw a change on only $29 \%$ of trials. This figure increased to $45 \%$ when a more liberal criteria was adopted, resulting in a main effect of block type, $F(1,18)=40$, $p<.0001, M S E=1 \%$. Observers were more likely to report a change when it involved one of the four rectangles closest to fixation $(M=55.5 \%)$ rather than one of the more peripheral rectangles $(M=31 \%), F(1,18)=28, p<.0001, M S E$ $=4 \%$. As expected, accuracy in aware trials was very high, and higher in the conservative block than in the liberal block, providing further evidence that different criteria for awareness were used (Conservative: $M=95 \%$; Liberal: $M=$ $85 \%), t(19)=5.1, p<.0001, S E M=1.6 \%$.

More importantly, examination of trials in which the observers reported being unaware of any change, indicate that the changing target was still selected at above chance levels for both the conservative instruction block $(M=$ $57 \%, t(19)=6.2, p<.0001, S E M=1.2 \%)$ and the liberal instruction block $(M=$ $55 \%, t(19)=3.3, p<.06, S E M=1.5 \%)$. Although this effect is numerically small, it is extremely robust, with 16 out of 20 observers in the liberal block and 19 out of 20 observers in the conservative block exceeding 50\% performance. As expected, correct selection was more frequent in the block with conservative instruction than in the one with liberal instructions, $F(1,18)=3.98, p<$ $.006, M S E=.5 \%$. Accuracy in the unaware trials was also generally higher for rectangles closest to fixation $(M=60.3 \%)$ than for those in more peripheral locations $(M=55.1 \%), F(1,18)=4.63, p<.045, M S E=1 \%$, a pattern that mimics the results for awareness.

Reaction time data also support the idea that even when observers fail to report a change, they nonetheless treat the change target differently from the 
distractor item. In general there was a strong trend for correct selection of the change target $(M=1039 \mathrm{msec})$ to be faster than selection of the distractor item $(M=1091 \mathrm{msec}), F(1,18)=3.91, p<.06, M S E=27315$. To further explore this effect, we ran separate analysis for peripheral items and central items. For peripheral rectangles, response time to a target $(\mathrm{M}=1083 \mathrm{msec})$ was $23 \mathrm{msec}$ faster than to a distractor $(M=1106 \mathrm{msec}), F(1,18)=5.93, p<.026, M S E=$ 1712. For items closest to fixation this RT benefit for targets $(M=995 \mathrm{msec})$ over distractors $(M=1076 \mathrm{msec})$ was even larger $(81 \mathrm{msec})$, although it failed to reach significance due to larger variability, $F(1,18)=2.5, p<.13, M S E=$ 52120 (medians for central locations are more variable because fewer trials contribute to them than to medians for peripheral locations).

One explanation for this overall pattern of results relies on the orienting of attention to the location of change. According to this view, the change of orientation automatically captures attention, possibly via a very weak residual motion transient. Once attention has been drawn even slightly towards the change target, it would favour its selection, leading to above-chance accuracy performance. In the trials in which the participant, contrary to this attention bias, decides to select the distractor, response time would be slow because attention would first need to be disengaged from the target location.

This attentional explanation has a precedent in the neuropsychology literature, where it has been proposed as a possible mechanism for "blindsight" (Cowey \& Stoerig, 1991), and it also receives support from studies that show orienting in the absence of awareness (McCormick, 1997). On the other hand, in standard change blindness studies there is no evidence for such an automatic capture of attention. Although it is possible that the active scanning of the scene (i.e. eye movements) exerts top-down control on attentional systems, and in this way neutralizes the weak exogenous orienting cues, this cannot be a complete explanation because change blindness studies that do not involve active scanning fail to show automatic capture of attention (e.g. Phillips, 1974; Simons \& Levin, 1997).

An alternative explanation assumes that representation of change can occur in the absence of attention. According to this view, the visual system is capable of tracking orientation change, but without attention, such representation s will not reach awareness and will not be accessible for explicit report. In Experiment 2 we attempt to distinguish between these two alternatives by using a spatial cueing paradigm.

\section{EXPERIMENT 2}

In Experiment 1, we showed above-chance performance in the selection of a target (changed item), even though participants reported being unaw are of such a change. One explanation for this behaviour would be that weak motion transients served as a cue, automatically drawing attention to the changed object, 
even though the change itself was not consciously perceived. If an unaware change automatically captures attention, then a subsequent probe at the location of change should be detected faster than a probe occurring opposite to the location of change (Posner, 1980; McCormick, 1997). Conversely, the lack of an orienting effect might suggest some form of automatic, pre-attentive representation of change.

To explore these possibilities we replicated the change blindness sequence used in Experiment 1, but replaced the $2 \mathrm{AFC}$ portion of the task with a speeded target discrimination in which a single target item appeared on the screen (see Figure 1c). Following discrimination of the target, participants indicated whether they had seen a rectangle change orientation by pressing the space bar. The nature of the target item was manipulated to explore the possibility that spatial cueing effects might vary as a function of cue-target similarity.

\section{Method}

Stimuli. The details of the change stimuli in the first two displays was identical to Experiment 1 (see Figure 1). The third display contained a single target item. For colour probe trials this target consisted of either a red or a green rectangle. The target was identical in size and orientation to the rectangle that had occupied the same location in the second display. For character probes, a single black ampersand $(\&)$ or percentage sign $(\%)$ appeared. These characters subtended approximately $0.5^{\circ} \times 0.5^{\circ} \mathrm{VA}$ and were centred on the position of the rectangle that had occupied the same location in the second display.

As before, a change was equally likely to occur at any one of the 16 possible locations, with each location hosting the change 16 times (for a total of 256 trials) in an order that was randomized for each participant. Of the 16 change trials at each location, 8 were followed by a target at the change location (valid cue) and 8 at the distractor location (invalid cue). The initial orientation of the changing rectangle and the identity of the probe were both equally distributed across the entire 256 trials in a different, random order for each participant.

Procedure. The general procedure and training regime was identical to Experiment 1 . During the third display, when only a single item was visible, the task of the participant was to identify the target as quickly as possible by pressing one of two marked keys. Unlike in Experiment 1, the aware response that followed target identification was a go/no-go response where the participant was instructed to press the space bar only when they were aware of a change. This modification was aimed at reducing interference with the primary response which now involved a two-choice key press rather than a mouse selection. In Experiment 2, change detection instructions were not manipulated. Instead a uniform "liberal" instruction was used in which participants were instructed to make an aware response if they either saw or thought they saw 
anything change in the first two displays. Participants completed a block of 256 trials with one type of probe, after which they were trained using the second type of probe, and completed a second 256-trial block. Block order was counterbalanced across participants.

\section{Results and Discussion}

As expected, levels of awareness in this experiment were very similar to those seen with the liberal criteria of Experiment 1. Overall awareness was $42 \%$ and there were no significant differences across types of probe (colour/character), types of cue (valid/invalid), eccentricity (near/far), or their interaction.

Of most interest in the current experiment is the pattern of response time as a function of cue validity and awareness. Figure 2 shows that when participants were aware of a change, they were faster to discriminate a subsequent target at that location $(M=684 \mathrm{msec})$ than at a distant distractor location $(M=701$ msec), $F(1,18)=4.7 p<.05, M S E=2371$. In contrast, there was no such effect

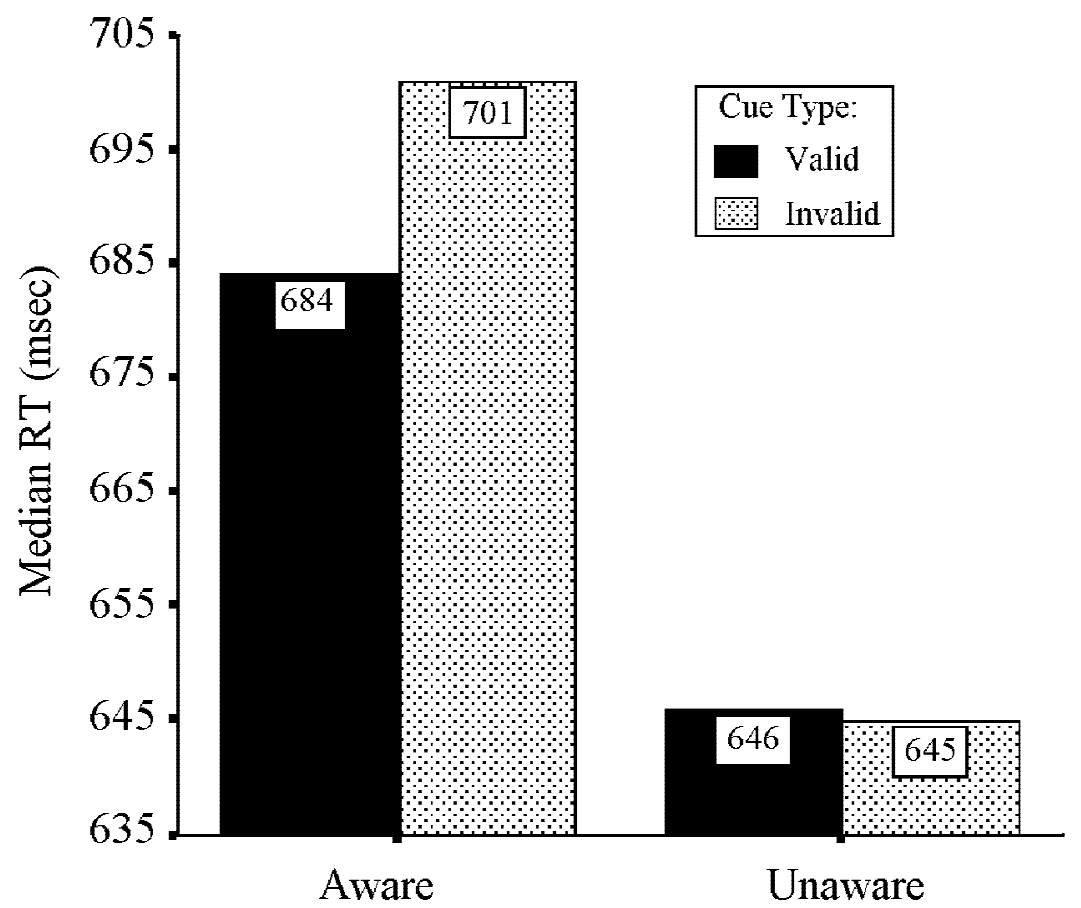

FIG. 2. Median reaction times (in $\mathrm{msec}$ ) for probes occurring at the location of change (valid location) and the distractor location (invalid location) for the two awareness conditions, in Experiment 2. There is a significant validity effect in the Aware but not in the Unaware condition. 
for unaware trials, with valid $(M=646 \mathrm{msec})$ and invalid cues $(M=645 \mathrm{msec})$ giving rise to very similar response latencies, $F(1,18)=.09$, ns, $M S E=1411$.

Colour targets $(M=607 \mathrm{msec})$ led to faster responses than character targets $(M=731 \mathrm{msec}), F(1,18)=57.8, p<.0001, M S E=21434$, and targets close to fixation $(M=663 \mathrm{msec})$ were discriminated faster than more distant ones $(M=$ $675 \mathrm{msec}), F(1,18)=10, p<.005, M S E=1244$, but these effects did not interact with cue validity. Target discrimination in aware trials $(M=692 \mathrm{msec})$ was slower than in unaware ones $(M=645 \mathrm{msec}), F(1,18)=16.9, p<.001, M S E=$ 10338 , suggesting that change detection or the response preparation triggered by change detection actively interferes with the discrimination task.

The lack of a validity effect when participants were unaware of the changes argues against the attentional hypothesis. On the other hand, the size of the validity effect when participants are aware of the change is a modest one (17 msec). Thus, the lack of a validity effect in the unaware trials may simply reflect the weakness or unreliability of the cue chosen to capture attention. Consistent with this view, unaware detection in Experiment 1 was only a modest effect. In the next two experiments we aimed at increasing the magnitude of this effect (Experiment 3 ) and the strength of change as an orienting cue (Experiment 4), to conceptually replicate the findings, and to test whether a potentially stronger orienting cue can lead to measurable unconscious orienting.

\section{EXPERIMENT 3}

In the current experiment we used an identical selection task to Experiment 1, but modified the display and certain aspects of the procedure in an attempt to increase effect size. There were three main modification s (see Figure 3). First, the number of rectangles was reduced from 16 to either 8 or 12 in separate blocks of trials, to examine the influence of set size. Second, the grid arrangement was replaced by a clockface design, to equate eccentricity for every item. Third, in the final display (Figure 3c) all items remained visible, but the change target and the distractor were marked by turning them from black to light grey. This maintained the global context and eliminated the large "off" transients which might have been disruptive in Experiments 1 and 2.

\section{Method}

Participants. Sixteen students participated in this study.

Stimuli. The stimuli objects were identical to those in Experiments 1 and 2. However, instead of 16 rectangles in a grid, there were now 8 or 12 objects (in separate blocks) organized as a ring or clockface. This latter change ensured that all items are equidistant from the central fixation point at approximately 


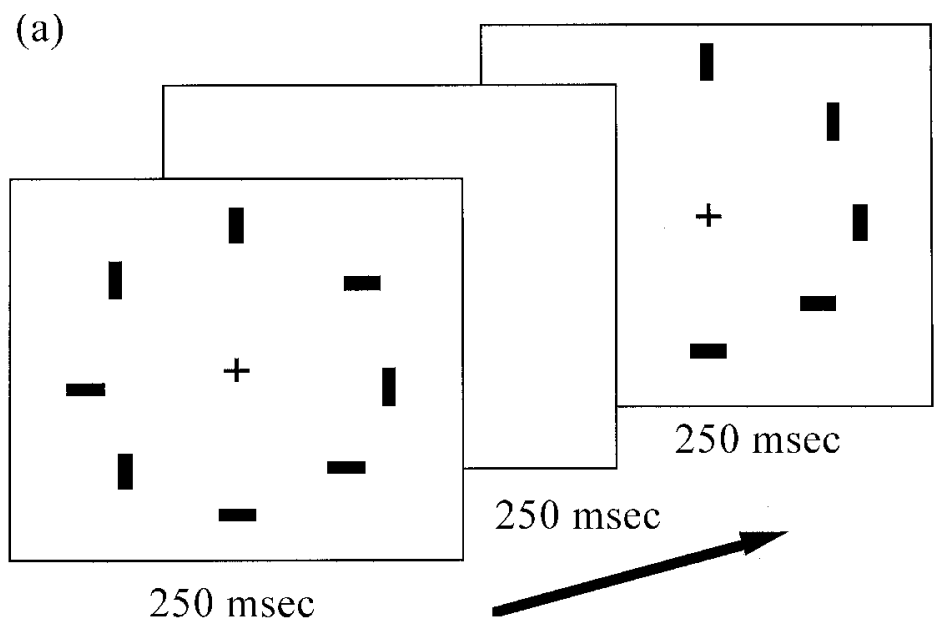

(b)

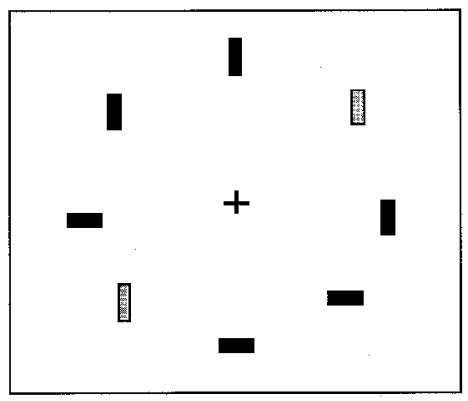

(c)

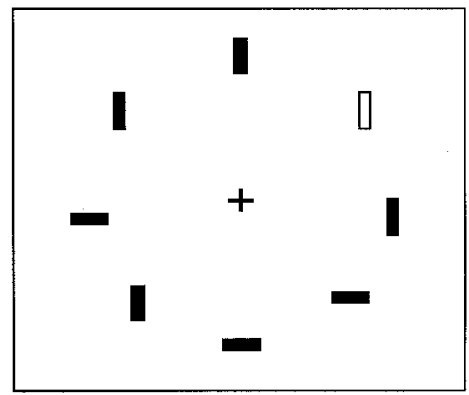

FIG. 3. Schematic representation of the layout and timeline for the stimuli used in Experiments 3 and 4. All displays were centred in the middle of the monitor screen without a visible border and the background and blank screen were grey rather than white. (a) The change detection sequence shown in the initial portion of each trial. The two rectangle frames are identical except for a single object which changes orientation during the blank ISI. Change was equiprobable at any location and here involves the rectangle located between 12 and 3 o'clock. In separate blocks, displays contained either 8 items (shown) or 12 items. The organization of the items was always in a ring and the 12-item display had the configuration of a standard clockface. (b) Experiment 3 response display. This followed the second change sequence display after a 0 ISI. Notice that all items now remain visible and change target and distractor are indicated by turning these items from black to light grey. Participants moved the central fixation point using the mouse and clicked on the rectangle that they thought most likely to have changed. The correct selection in this case would be the rectangle located between 12 and $3 \mathrm{o}$ ' clock. (c) Experiment 4 response display. This followed the second change sequence display after a 0 ISI. A target item appeared which was always one of two coloured rectangles (red or green, shown in white here for display purposes). Participants made a speeded response using one of two marked keys. The position of the target could overlap either with the change location (valid cue, shown) or the distractor location (invalid cue). 
$4.6^{\circ}$. Figure 3 shows the layout for an 8 -item display; the twelve-item display was organized as a standard clockface.

As in Experiment 1, a change could occur with equal probability at any one of the locations but each object played host to the change for 28 rather than 16 trials, giving a total of 224 and 336 trials, for the eight- and twelve-item displays respectively. Unlike in Experiment 1, the orientation of the change target and the distractor item was constrained. That is, each target was horizontal on 14 trials and vertical on 14 trials and the distractor item orientation was congruent with the target on 14 trials and incongruent on 14 trials. These factors varied randomly across all trials as in Experiment 1.

A more important change in this new experiment concerned the third display (see Figure 3b). Rather than removing the non-relevant rectangles, the target and distractor items were identified by changing their colour from black to light grey. All items remained on the screen until the participant made a response.

Procedure. The procedure was essentially the same as for Experiment 1, with the exception that there was no manipulation of awareness instructions. As in the liberal criterion condition used in Experiments 1 and 2, participants were told to indicate an aware response (by pressing one of two marked keys) based on whether they saw or thought they saw anything change in the first two displays. Participants completed one block of either 8- or 12-item trials, after which they were trained using the second type of display, and completed a second block of trials. Order was counterbalanced across participants.

\section{Results and Discussion}

The set-size manipulation had a strong effect on awareness responses. In the 8 -item blocks, participants reported being aware of a change on $52 \%$ of the trials. This fell to $40 \%$ with 12 items, leading to a significant main effect of block, $F(1,15)=24, p<.0001, M S E=.5 \%$. As expected, selection of the target was very accurate in the aware trials $(M=89 \%)$.

Analysis of the unaware trials replicated the finding of Experiment 1, with performance levels exceeding chance in both 8-item blocks $(M=63 \%)$, and 12 item-blocks $(M=58 \%), t \mathrm{~s}(15)=6.0$ and 5.3, respectively, $p \mathrm{~s}<.0001, S E M=$ $2 \%$. This effect was also highly consistent, appearing in the performance of 14 out 16 participants in the 12 -item block, and in all 16 participants in the 8 -item block. In Experiment 1, the reaction time data suggested sensitivity to change in unaware trials, and this same pattern is apparent here, with correct unaware selections $(M=1127 \mathrm{msec})$ being significantly faster than incorrect ones $(M=$ $1176 \mathrm{msec}), F(1,14)=18, p<.001, M S E=2073$. 
Could this unaware detection effect be caused by some form of strategic response bias? In particular, above-chance performance could occur if participants monitored only half of the display and assumed that when a change was not detected it must have occurred in the opposite location.

A close inspection of the data reveals that it is very unlikely for such a strategy to be responsible for the observed effect. First, as set size decreases, both awareness and unaware detection increase, a pattern that cannot be accounted for in terms of the previously mentioned strategy. Second, such a strategy would not predict and cannot explain the differences in response times that were observed for correct and incorrect selection during unaware trials. Third, the use of such a strategy should lead to a pattern of correlations between awareness for a given item and unaware detection of that item and its opposite distractor. Specifically, for a given item, awareness should be positively correlated with unaware detection in the opposite item and inversely correlated with unaware detection in the same item ${ }^{2}$. Such a pattern is not present in our data. Finally, given the consistency of the present findings, the same strategy would have to have been adopted by all 16 participants, a possibility made even more unlikely given that debriefing reports never once indicated such strategy use. Taken together these factors argue against such a response bias explanation.

We return now to the question of whether attentional or non-attentional representations might account for the unaware detection effect of Experiments 1 and 3.

\section{EXPERIMENT 4}

In Experiment 2, we found a validity effect only for changes that were accompanied by conscious awareness. One possibility for the lack of an unaware orienting response was the limited strength of the basic change signal. After all, this change signal had only led to modest (i.e. 5\%) above chance unaw are performance in Experiment 1. In Experiment 3, we boosted this effect up to 13\% by changing the layout of the display and reducing the number of target items. Here, we make similar changes to the display used in Experiment 2, to examine whether an increase in unaware change strength would also be reflected in patterns of attentional cueing.

${ }^{2}$ Examination of these patterns across each item for each participant revealed no correlation between awareness and unaware detection in the opposite location $(r=-.006, \mathrm{~ns})$, and a slight positive correlation between awareness and unaware detection at the same location $(r=.16, p<.001)$. 


\section{Method}

Participants. Sixteen students participated in this experiment.

Stimuli. The change sequence stimuli were identical to that used in Experiment 3. Also, as in Experiment 3, rather than removing the non-relevant rectangles in the third display, all items remained from the second display, but the colour of the target item changed from black to either red or green. The nature of the target discrimination was not manipulated in this experiment and targets were always colour defined. We retained the colour probes because in Experiment 2 colour probes yielded larger orienting effects than character probes (Colour, $M=22.5 \mathrm{msec}$; Character, $M=11 \mathrm{msec}$ )

Procedure. The procedure was identical to that used in Experiment 2. Participants completed separate blocks of 8- or 12-item trials, in a counterbalanced order.

\section{Results and Discussion}

As in Experiment 2, there was a significant validity effect for aware trials (Valid, $M=570$; Invalid, $M=594, F(1,14)=15, p<.002, M S E=600$ ) but not for unaware trials (Valid, $M=559$; Invalid, $M=562, F(1,14)=.74$, ns, $M S E=$ 303). That is, while the current set-size manipulations appeared to increase aware orienting (relative to Experiment 2) and had a strong influence on the general level of awareness (8-item, $M=49 \%$; 12 -item, $M=39 \%, F(1,14)=$ $28.8, p<.0001, M S E=.5 \%)$ there was still no indication that attention was being drawn to change locations in the unaware trials (see Figure 4).

\section{GENERAL DISCUSSION}

In line with previous change blindness studies, the four experiments reported here demonstrate that observers often find it difficult to consciously perceive changes in visual displays under circumstances in which local transients are reduced or removed (e.g. Phillips, 1974; Rensink et al., 1997). Even though observers were expecting changes, explicit detection rates were very poor, rarely exceeding 50\%. However, in Experiments 1 and 3 we showed that when presented with a forced choice task, observers were still able to select the changed item at above chance levels even when they report being unaware of any change. Although there have been many previous reports of perception without awareness in the cognitive literature (see Chen, 1998; Mack \& Rock, 1998; Moore \& Egeth, 1997; Shapiro, Driver, Ward, \& Sorenson, 1997 for 


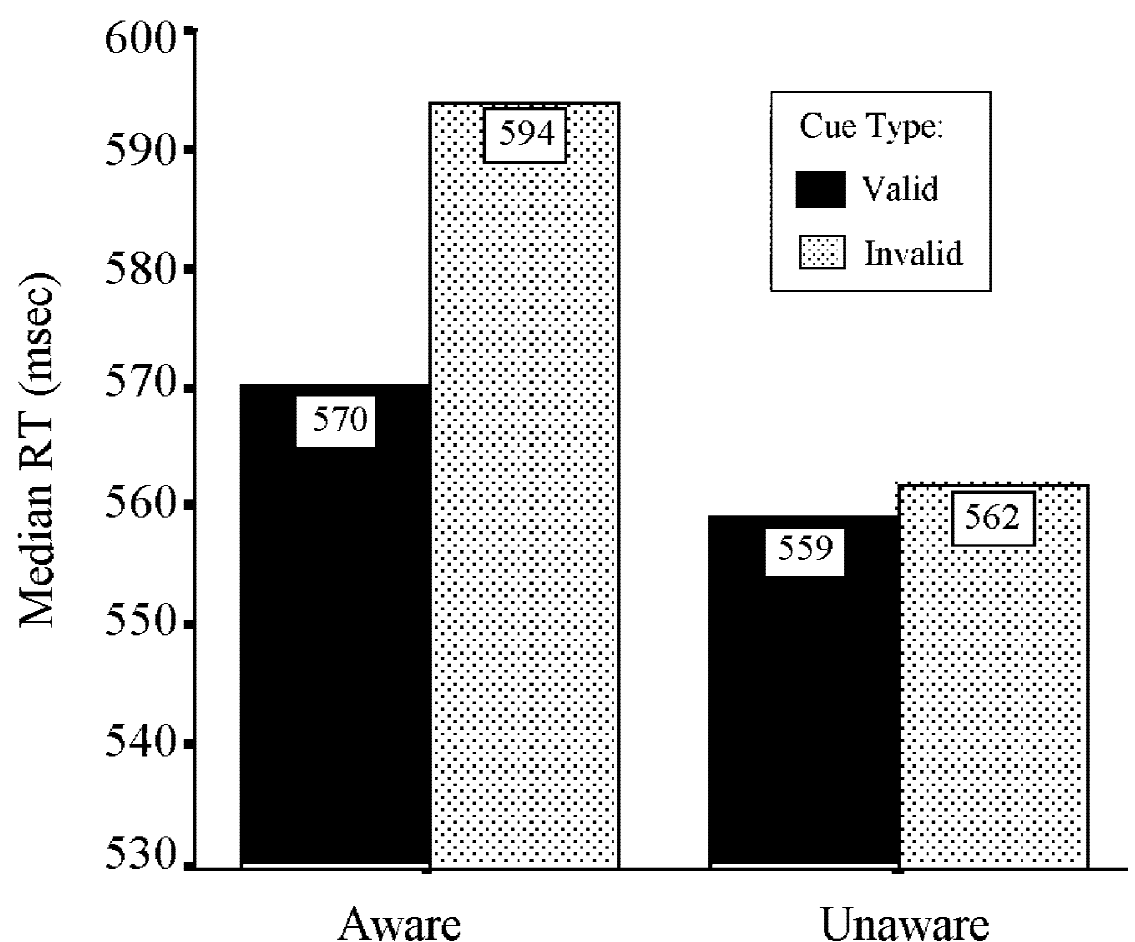

FIG. 4. Median reaction times (in $\mathrm{msec}$ ) for probes occurring at the location of change (valid location) and the distractor location (invalid location) for the two awareness conditions, in Experiment 4. There is a significant validity effect in the Aware but not in the Unaware condition.

recent examples) we believe this is the first example to demonstrate unconscious detection of change over time.

In Experiments 2 and 4, we used a spatial cueing paradigm to investigate whether such unconscious change detection might have its origins in some form of below-threshold shift of attention. Although the orientation change contained in our displays proved to be a good spatial cue when observers were aware of the change, we found no evidence of a validity effect when changes were not explicitly detected. As illustrated in Figures 2 and 4, this difference between aware and unaware trials is not simply a magnitude effect: The unaware data are basically flat. Although it is always difficult to build strong conclusions based solely on null results, the complete lack of unaware reaction time modulation in either experiment, even when aware orienting had been considerably boosted by our display modifications (Experiment 4), taken together with the knowledge that such unaware modulations are 
possible (McCormick, 1997), gives us some confidence that covert allocation of attention is not a major factor in the detection results of Experiments 1 and $3 .^{3}$

How can participants correctly detect the location of the change, given that they show no evidence of orienting to it? One possibility is that a diffuse distribution of attention over the visual field helps to represent the object transformation, but in such a weak manner that the representation fails to reach awareness and fails to trigger an orienting response. One way to explore this issue would be to try to develop a test of attentional allocation that is more sensitive than the current orienting paradigm. An alternative, and probably more feasible, approach would be to introduce some form of secondary task at fixation. If such a task were attentionally demanding (e.g. detecting a specific letter in a rapidly presented stream of letters and digits) then, according to the previous explanation, the unaware detection effect should disappear.

Another explanation, already touched on in Experiment 3, is the idea of a strategic response bias. That is, participants preferentially attend to one side of the display, assuming that when they don't see a change, it must have occurred on the other side. Although our results make this a very unlikely explanation, they do not rule out a strategy in which participants monitor different parts of the display during different parts of the experiment. In its most extreme form, a participant may choose randomly, on a trial by trial basis, which items to monitor. ${ }^{4}$

Even in the absence of an explicit strategy on the part of an observer, it is still possible that a response could be guided by information about what has not changed, rather than information about what has changed. Such a situation could arise if an observer were somehow able to retrospectively access those items that were being held or selected by attention on a given trial-a variant of the so-called "labelled line" hypothesis (e.g. De Valois \& De Valois, 1993). If the changed item had been selected by attention then an aware response would be predicted. If neither of the two probe items had been selected by attention then performance should be at chance. If, however, the non-changing probe item was being held by attention then the observer may guess that the opposite

${ }^{3}$ McCormick's study differed from the current work in using a sub-threshold object onset as a cue. In contrast, our experiments relied on a supra-threshold object transformation. Clearly, the processes involved in setting up a new representation of an object are likely to be different from those involved in transforming an existing object (Kahneman et al., 1992). It is thus possible that there is a quantitative rather than a qualitative difference involved here, with our change cue simply being too weak to survive in the absence of awareness.

Including catch trials alone would not rule out the existence of such a strategy. For example, no bias in the response pattern to catch trials would be evident if the subject's strategy was to monitor half of the screen but change randomly which half was monitored at any given time. 
probe item was changing simply because they are certain that the attentionally selected item did not change.

Another alternative explanation, and one of the most common criticisms of studies reporting unconscious effects, is the potential contamination of conscious (aware) processes into what is being labelled unconscious (unaware). That is, on some fraction of trials participants may simply mislabel an aware response as an unaware response, either due to uncertainty or error, leading to artificially inflated unaware performance. Similar contamination could also occur if our $2 \mathrm{AFC}$ procedure were simply a more sensitive test of aw are change detection than more standard explicit reporting methods. The finding of above-chance unaware detection in Experiment 1 under both highly conservative as well as liberal awareness criterion goes some way to rebutting this explanation.

More convincing, is the lack of an orienting effect in Experiments 2 and 4, despite the use of the same awareness criterion as that used in Experiments 1 and 3. That is, if the detection effect were spurious, a consequence of aware trials being reported as unaware ones, then the same should be expected in the orienting experiments: Mislabelling some aware trials (which show a validity effect) should lead to an artificial "unaware" validity effect. The fact that this did not happen suggests either that contamination is highly sensitive to task context or that it is not operating in these studies. Similarly, if the unaware detection effect were simply due to the $2 \mathrm{AFC}$ task being more sensitive than the current aware response measure, then, by the same logic, we should predict an orienting effect in Experiments 2 and 4 as RT measures are usually thought to be as, if not more, sensitive than accuracy measures.

${ }^{5}$ While such an explanation relies heavily on a number of assumptions-about our ability to retrospectively access attentionally selected items, the number of items selected in a given display and the extent to which we could use such marked items in making decisions-initial attempts to model our data using such an analysis suggests that this alternative warrants further investigation. For example, if we assume an $\mathrm{N}$-item display, and further assume that attention can only select A items in any given trial, then the probability of the non-changing probe item being selected by attention during a given trial can be expressed as $\mathrm{P}_{\text {held }}=\mathrm{A} / \mathrm{N}-1$, while the probability of such an item not being selected can be expressed as $\mathrm{P}_{\text {not held }}=(\mathrm{N}-1)-\mathrm{A} / \mathrm{N}-1$. The use of $\mathrm{N}-1$ here indicates that we are concerned only with cases in which attention has not selected the one item in each display that actually does change. Let's further assume that a correct selection will be made when the non-changing probe item is selected by attention (i.e. probability of a correct response $K=1.0$ ) while correct selection will be at chance when neither probe is attended (i.e. correct response probability $\mathrm{U}=.5)$. Given these parameters, the probability of making a correct response can be expressed as $\mathrm{P}_{\text {correct }}=\left(\mathrm{P}_{\text {held }} \times \mathrm{K}\right)+\left(\mathrm{P}_{\text {not held }} \times \mathrm{U}\right)$, where $\mathrm{A}=2, \mathrm{~K}=1.0$, and $\mathrm{U}=.5$. This formula provides a close fit to the results of both Experiment 1 (predicted $=56 \%$, actual conservative $=55 \%$, actual liberal $=57 \%$ ) and Experiment 3 (predicted 12 items $=59 \%$, actual $=58 \%$; predicted 8 item $=64 \%$, actual $=63 \%$ ). Although goodness of fit here is highly dependent on parameter selection, this model may prove to be a useful tool in exploring the nature of the unaware detection effect. We thank Ron Rensink for pointing out this analysis. 
One way to address this issue, an approach that would also be useful in regard to possible strategic response biases, would be to try to identify neural or physiological markers of change detection in the absence of awareness. ${ }^{6}$ Another possibility would be to find convergent evidence for unaware detection using tasks other than the 2AFC. Although forced choice tasks have been used with great success in previous work probing unconscious visual experience (e.g. blindsight), and here has the advantage of providing some measure of change localization, the use of other measures of unaware performance, such as those used to explore priming, might reduce the problems of conscious contamination, response bias, or unequal sensitivity (Shapiro, this issue).

Other theoretically more interesting explanations for unaware detection would need to postulate some form of non-attentional representational system capable of registering change in the absence of awareness. Perhaps the displays in the current studies are sparse enough and/or regular enough for a layout system to automatically compute a mismatch between two views (see e.g. Rensink, this issue). Alternatively, enhanced performance without awareness might reflect the involvement of systems designed to process other aspects of visually guided behaviour, such as locating, reaching, and grasping objects (Chun \& Nakayama, this issue; Goodale \& Milner, 1992). Such systems are thought to be subserved by neural mechanisms that are relatively independent from those subserving visual recognition and perceptual awareness.

Clearly, the current work represents a first step in exploring the nature of unaware change detection. Future studies will need to address both the methodological concerns we have discussed here and also will need to establish that such unaware detection is observable under more ecologically valid conditions, such as in naturalistic complex scenes, as well as in dynamic environments where the observer or other objects are in motion (e.g. Wallis \& Bülthoff, this issue). If such studies were to confirm our prediction, that representational systems capable of signalling change are operating outside the realm of attention, then this would pose a serious challenge to the strict link between attention and spatio-temporal coherence, a link that has become a central theme in many current theories of scene perception (e.g. Rensink, this issue).

Specifically, previous studies have shown that attention is not sufficient to guarantee change detection and that people sometimes fail to consciously detect changes to objects that are at the centre of attention (Levin \& Simons, 1997; O' Regan et al., this issue). Our findings suggest that attention is not always necessary for the representation of change. Although conscious visual experience of change may indeed require attention, the current work raises the

${ }^{6}$ A technique particularly suitable for this approach would be Event Related Potentials (ERPs), because ERPs allow post-test comparisons based on the type of response such as correct/error and aware/unaware (e.g. Luck, Vogel, \& Shapiro, 1996). Examination of eye-movement records may also be very useful (e.g. Hayhoe, Bensinger, \& Ballard, 1998). 
possibility that our overall mental representation of the visual world is not as incomplete, incoherent, or as dependent on the last attentional act as has sometimes been suggested (e.g. Horowitz \& Wolfe; Rensink, this issue, 1998).

Finally, it seems relevant to ask why unaware detection of change, if it exists, fails to assist aware or explicit detection in standard change blindness tasks? A recent report claims that some observers, but not others, do appear to "sense" reliably that something is changing in the scene, often several seconds before they can consciously locate or identify the change (Rensink, 1998). The finding of a lag between initial representation and subsequent explicit report is all the more interesting given large individual differences in sensitivity. Change information might always be available, but only "sensitive" tests, as in the current experiments, or "sensitive" observers, as in Rensink' s report, may be able to express it. Alternatively, unconscious processes might be truly unable to assist voluntary action, and might only be expressed when subjects are forced to make a response (Block, 1995).

In summary, consistent with previous findings, our observers were unaware of many of the changes which occurred in their visual environment and those changes did not appear to capture attention. However, those changes did influence some aspects of performance, suggesting that explicit measures of detection could be underestimating the representation of change in the visual system. Our findings raise questions about the relationship between attention and the representation of change and in so doing, would seem to pose problems for models of scene perception that strictly limit spatio-temporal coherence to conditions of focused attention. It seems likely that focused attention can serve not only to originate representations of change but also to modulate representations that originate elsewhere in the visual system.

\section{REFERENCES}

Bar, M., \& Biederman, I. (1998). Subliminal visual priming. Psychological Science, 9,(6), 464-469.

Block, N. (1995). On a confusion about a function of consciousness. Behavioral and Brain Sciences, 18 (2), 227-287.

Bridgeman, B., Hendry, D. \& Stark, L. (1975). Failure to detect displacements of the visual world during saccadic eye movements. Vision Research, 15, 719-722.

Chen, Z. (1998). Inattentional amnesia: Evidence of perception without awareness. Investigative Ophthalmology and Visual Science, 39(4), S629.

Chun, M.M., \& Nakayama, K. (this issue). On the functional role of implicit visual memory for the adaptive deployment of attention across scenes. Visual Cognition, 7, 65-81.

Cowey, A., \& Stoerig, P. (1991). Reflections on blindsight. In A.D. Milner, \& M.D. Rugg, (Eds.), The neuropsychology of consciousness (pp. 11-39). London: Academic Press.

DeSchepper, B., \& Treisman, A. (1996). Visual memory for novel shapes: Implicit coding without attention. Journal of Experimental Psychology: Learning, Memory and Cognition, 22(1), $27-47$. 
De Valois, K.K., \& De Valois, R.L. (1993). Spatial channels and labeled lines. In R. Shapley \& D. Lam (Eds.), Contrast sensitivity (pp. 231-249). Cambridge MA: MIT Press.

Driver, J., Baylis, G.C., \& Rafal, R.D. (1992). Preserved figure-ground segregation and symmetry perception in visual neglect. Nature, 360, 73-75.

Goodale, M.A., \& Milner, A.D. (1992). Separate visual pathways for perception and action. Trends in Neurosciences, 15(1), 20-25.

Graves, R.E., \& Jones, B.S. (1992). Conscious visual perceptual awareness vs. non-conscious visual spatial localisation examined with normal subjects using possible analogues of blindsight and neglect. Cognitive Neuropsychology, 9(6), 487-508.

Grimes, J. (1996). On the failure to detect changes in scenes across saccades. In K. Akins (Ed.), Vancouver studies in cognitive science: Vol. 5. Perception, (pp. 89-109). New York: Oxford University Press.

Hayhoe, M. (this issue). Vision using routines: A functional account of vision. Visual Cognition, 7, 43-64.

Hayhoe, M., Bensinger, D., \& Ballard, D.H. (1998). Task Constraints in visual working memory. Vision Research, 38, 125-137.

Henderson, J.M. (1997). Transsaccadic memory and integration during real-world object perception. Psychological Science, 8, 51-55.

Horowitz, T.S., \& Wolfe, J.M. (1998). Visual search has no memory. Nature, 394, 575-577.

Kahneman, D., Treisman, A.M., \& Gibbs, B. (1992). The reviewing of object files: object-specific integration of information. Cognitive Psychology, 24, 175-219.

Kolb, F.C., \& Braun, J. (1995). Blindsight in normal observers. Nature, 377, 336-338.

Levin, D.T., \& Simons, D.J. (1997). Failure to detect changes to attended objects in motion pictures. Psychonomic Bulletin and Review 4(4), 501-506.

Luck, S.J., Vogel, E.K., \& Shapiro, K.L. (1996). Word meanings can be accessed but not reported during the attentional blink. Nature, 382, 616-618.

Mack, A., \& Rock, I. (1998). Inattentional blindness. Cambridge, MA: MIT Press.

Marcel, A.J. (1983a). Conscious and unconscious perception: Experiments on visual masking and word recognition. Cognitive Psychology, 15, 197-237.

Marcel, A.J. (1983b). Conscious and unconscious perception: An approach to the relations between phenomenal experience and perceptual processes. Cognitive Psychology, 15, 238-300.

McCormick, P.A. (1997). Orienting attention without awareness. Journal of Experimental Psychology: Human Perception and Performance, 23(1), 168-180.

Milner, B., Corkin, S., \& Teuber, H. (1968). Further analysis of the hippocampal amnesic syndrome: 14 year follow-up study of H.M. Neuropsychologia, 6, 215-234.

Moore, C.M., \& Egeth, H. (1997). Perception without attention: Evidence of grouping under conditions of inattention. Journal of Experimental Psychology: Human Perception and Performance, 23(2), 339-352.

O' Regan, J.K., Deubel, H., Clark, J.J., \& Rensink, R.A. (this issue). Picture changes during blinks: Looking without seeing and seeing without looking. Visual Cognition, 7, 191-211.

Pashler, H. (1988). Familiarity and visual change detection. Perception and Psychophysics, 44, 369-378.

Pelli, D.G., \& Zhang, L. (1991). Accurate control of contrast on microcomputer displays. Vision Research, 31, 1337-1360.

Phillips, W.A. (1974). On the distinction between sensory storage and short-term visual memory. Perception and Psychophysics, 16, 283-290.

Posner, M.I. (1980). Orienting of attention. Quarterly Journal of Experimental Psychology, 32, $3-25$.

Rensink, R.A. (1990). Toolbox-based routines for Macintosh timing and display. Behavior Research Methods, Instruments, and Computers, 22(2), 105-117. 
Rensink, R.A. (1998). Mindsight: Visual sensing without seeing. Investigative Ophthalmology and Visual Science, 39(4), S631.

Rensink, R.A. (this issue). The dynamic representation of scenes. Visual Cognition, 7, 17-42.

Rensink, R.A., O' Regan, J.K., \& Clark, J.J. (1997). To see or not to see: The need for attention to perceive changes in scenes. Psychological Science, 8, 368-373.

Rensink, R.A., O' Regan, J.K., \& Clark, J.J. (this issue). On the failure to detect changes in scenes across brief interruptions. Visual Cognition, 7, 127-145.

Ro, T., \& Rafal, R.D. (1996). Perception of geometric illusions in hemispatial neglect. Neuropsychologia , 34(10), 973-978.

Shapiro, K.L. (this issue). Change blindness: Theory or paradigm? Visual Cognition, 7, 83-91.

Shapiro, K.L., Driver, J., Ward, R., \& Sorenson, R.E. (1997). Priming from the attentional blink: A failure to extract visual tokens but not visual types. Psychological Science, 8(2), 95-100.

Simons, D.J. (1996). In sight, out of mind: When object representations fail. Psychological Science, 7, 301-305.

Simons, D.J., \& Levin, D.T. (1997). Change blindness. Trends in Cognitive Sciences, 1(7), 261-267.

Steinman, S.B., \& Nawrot, M. (1992). Real-time color-frame animation for visual psychophysics on the Macintosh computer. Behavior Research Methods, Instruments, and Computers, 24(3), 439-452.

Treisman, A. (1988). Features and objects: The fourteenth Bartlett memorial lecture. Quarterly Journal of Experimental Psychology, 40A(2), 201-237.

Wallis, G., \& Bülthoff, H. (this issue). What's scene and not seen: Influences of movement and task upon what we see. Visual Cognition, 7, 175-190.

Warrington, E.K., \& Weiskrantz, L. (1968). New method for testing long-term retention with special reference to amnesic patients. Nature, 217, 972-974.

Weiskrantz, L. (1986). Blindsight: A case study and implications. Oxford, UK: Oxford University Press. 